\title{
Evolutionary design on a budget: robustness and optimality of bacteriophage T7
}

\author{
L. You and J. Yin
}

\begin{abstract}
Exploring how biological systems have been 'designed' by evolution to achieve robust behaviours is now a subject of increasing research effort. Yet, it still remains unclear how environmental factors may contribute to this process. This issue is addressed by employing a detailed computer model for the intracellular growth of phage T7. More than 150000 in silico T7 mutants were generated and the rates and efficiencies of their growth in two host environments, namely, a realistic environment that offered finite host resources for the synthesis of phage functions and a hypothetical environment where the phage was supplied infinite host resources, were evaluated. Results revealed two key properties of phage T7. First, T7 growth was overall robust with respect to perturbations in its parameters, but fragile with respect to changes in the ordering of its genetic elements. Secondly, the wild-type T7 had close to optimal fitness in the finite environment. Furthermore, a strong correlation was found between fitness and growth efficiency in the finite environment. The results underscore the potential importance of the environment in shaping robust design of a biological system. In particular, the strong correlation between fitness and growth efficiency suggests that $\mathrm{T} 7$ may have evolved to maximise its growth rate by minimising waste of finite resources.
\end{abstract}

\section{Introduction}

Recent computational and experimental studies have begun to reveal system-level properties of diverse cellular systems $[1-10]$. An often characterised property is the robustness of these systems, which can be defined as the stability of a phenotype in the presence of genetic and environmental variations [11].

Using a detailed kinetic model, Barkai and Leibler analysed how the output of a chemotaxis network would respond to perturbations in the kinetic parameters that define the network behaviour [12]. Their simulations demonstrated that some properties of the network, such as precision of adaptation, are robust to parametric perturbations. Key findings of this computational study were later verified by experiments [13]. They also noted that not all properties are robust to all parameters. For example, the adaptation time and the steady-state tumbling frequency of the network were found to be sensitive to perturbations in parameters [12]. From a system control perspective, Yi et al. [14] showed that the robustness of the chemotaxis network can be attributed to an integral feedback loop embedded in the network. It has been argued that the robustness might be a generic feature necessary to ensure proper functioning of a wide variety of biological systems [12]. This argument was extended by Morohashi et al. [15] who suggested that the robustness

\section{(C) IEE, 2006}

IEE Proceedings online no. 20050026

doi:10.1049/ip-syb:20050026

Paper first received 19th May and in revised form 2nd December 2005

L. You is with the Department of Biomedical Engineering and Institute for Genome Sciences and Policy, Duke University, Durham, NC 27708, USA

J. Yin is with the Department of Chemical and Biological Engineering, University of Wisconsin-Madison, 1415 Engineering Drive, Madison, WI 53706-1691, USA

E-mail: you@duke.edu to variations could be used as a measure of the plausibility of the models of a given system.

These arguments have gained support from recent modelling and experimental studies. A network model for segmentation in Drosophila demonstrated robust features: the model was able to predict the correct segmentation pattern for a wide range of parameter settings [16]. The same group later found that another system - the Drosophila neurogenic network - also demonstrated significant robustness with respect to network parameters [17]. Furthermore, computational [18-20] and experimental [21] studies have suggested that circadian clocks often demonstrate robust behaviour with respect to intrinsic or external perturbations. From another perspective, robust performance has become a central design goal of synthetic gene circuits, where efforts are being made to counteract the effect of cellular noise on circuit function [22], for example, using negative feedback control [23] or cell-cell communication $[24,25]$.

In addition to intrinsic organisation of a cellular network, the environment may play a role in determining the performance of the network. It has been established that there is complex interplay between the genotype of an organism and its environment [26, 27]. Computational analyses have provided insight into the understanding of such interactions at the cellular level. The demand theory proposed by Savageau $[28,29]$ suggests that bacterial operons regulated by repressors are selected for in low-demand environments, whereas those regulated by activators are selected for in high-demand environments [7, 28]. These predictions have been found to agree well with experimental observations [29]. In addition, recent work suggests that the evolution of gene expression in the yeast Saccharomyces cerevisiae is constrained by associated cost of energy consumption [30]. Complementary to these, we showed by simulation that growth environments of an organism can significantly impact the nature and degree of the genetic interactions among different deleterious mutations [31]. 
By simulating and characterising growth of hundreds of thousands of in silico T7 mutants, we show here that the environment may play an important role in shaping not only the activities of discrete biochemical functions, but also the regulatory network of interactions that underlie the development of an organism. Specifically, we examine effects of different perturbations in the context of two growth environments: one corresponds to a realistic host cell that has finite resources and the other represents a hypothetical host cell with infinite resources. Our results indicate that the wild-type T7 is close to optimal in terms of its growth rate in the finite-resource environment but not in the infinite environment. Furthermore, our results suggest that $\mathrm{T} 7$ possesses a 'robust-yet-fragile' property, a common feature of biological and advanced engineering systems [32-34].

\section{Materials and methods}

\subsection{Phage T7 model}

By accounting for and incorporating existing experimental data and mechanisms, Endy et al. [35] developed a genetically structured model to simulate the infection of a Escherichia coli cell by a wild-type T7 particle. The current model recasts previous versions using an objectoriented approach [36]. It treats the genome as an array of 74 genetic elements, where each element consists of one or more overlapping genes, promoters, transcription terminators, RNase III processing sites and spacer DNAs. It accounts for the mechanisms of the entry of T7 DNA into the host cell, synthesis of T7 mRNAs and proteins, regulation of $\mathrm{T} 7$ gene expression, degradation of host DNA and replication of T7 DNA, assembly of procapsids and formation of $\mathrm{T} 7$ progeny. It also extends earlier versions by accounting for the stoichiometric relation of the $\mathrm{T} 7 \mathrm{heli-}$ case/primase (gp4A) and the DNA polymerase (gp5) in forming replication complexes, or replisomes, as well as the stoichiometric balance between the number of replication complexes and the maximum number of replication forks that can form on the newly synthesised $\mathrm{T} 7$ genomes. In addition, it allocates E. coli RNA polymerases (EcRNAPs) and T7 RNA polymerases (T7RNAPs) to the synthesis of different mRNAs based on the relative activities of the promoters. Further, the current model has been sufficiently generalised to enable simulation of the growth of T7 mutants with re-ordered genomes [37] and to account for effects of host physiological state on $\mathrm{T} 7$ growth [36]. Detailed implementation of the model is described in Supplemental Online Materials (SOM), adapted from You [38]. The computer code for the model is available at http://www.duke.edu/ you. Analysis of the simulation output was done in MATLAB (MathWorks, Inc.).

\subsection{Fitness and growth efficiency}

We define fitness $(F)$ as the maximum production rate of progeny during T7 intracellular growth, as follows

$$
F \equiv \max \left(\frac{\text { number of phage particles }}{\text { time after infection }}\right)_{t}
$$

where the subscript $t$ indicates that the maximum value was taken over the entire course of simulation. This simple metric quantifies the effectiveness of a given $\mathrm{T} 7$ variant in producing progeny during one cycle of infection. $F$ always reaches maximum at the time point where the number of progeny reaches maximum (data not shown). It is an appropriate measure of $\mathrm{T} 7$ fitness, if $\mathrm{T} 7$ lyses the host cell at this time point. Under most conditions, it increases with several other metrics such as rise rate [36], maximum doubling rate [37] or burst size [31]. For instance, if all T7 variants reach their maximum number of progeny at the same time point, the maximum growth rate will be proportional to the burst size. Each metric is more appropriate in measuring the potential 'success' of a T7 variant in different settings, but use of these alternatives does not change the main conclusions of this article.

To further access effects of environments, we define the efficiency $(E)$ of $\mathrm{T} 7$ growth by

$E \equiv \max$

$\left(\frac{\text { amino acids incorporated into progeny }}{\text { amino acids incorporated into particle proteins }}\right)_{t}$

where the subscript $t$ has the same meaning as in (1). We have focused on only one aspect of growth efficiency: the above function measured how efficiently the virus was able to balance the production of different viral proteins. In a 'perfectly' efficient virus, all particle proteins will be produced following the stoichiometry by which they are incorporated in the progeny (Table S5). Deviation from this stoichiometry will result in 'waste' of resources and thus decrease the efficiency. Similar efficiency metrics can be defined for other processes, for example, efficiency in the consumption of energy or metabolites [39].

\subsection{In silico mutations}

Every in silico T7 mutant contains one of the following two types of perturbations: changes to one or more parameters that define T7 physiology or reordering of genetic elements along the $\mathrm{T} 7$ genome. In the former case, up to 30 parameters were varied within a range from 0.1 to 10 times their base values while the other parameters were kept constant. These parameters included relative activities of six EcRNAP promoters (A1, A2, A3, B, C and E), relative activities of 15 T7RNAP promoters $(1.1 \mathrm{~A}, 1.1 \mathrm{~B}, 1.3,1.5$, $1.6,2.5,3.8,4 \mathrm{C}, 4.3,4.7,6.5,9,10,13$ and 17), the T7RNAP elongation rate $\left(k_{\mathrm{PT} 7}\right)$, the T7 DNA polymerase elongation rate $\left(k_{\mathrm{PD}}\right)$, the $\mathrm{T} 7$ procapsid assembly rate constant $\left(k_{\mathrm{a}}\right)$, the T7 DNA packaging rate $\left(k_{\text {pack }}\right)$, the gp0.7EcRNAP association constant $\left(K_{1}\right)$, the gp2-EcRNAP association constant $\left(K_{2}\right)$, the T7RNAP-gp3.5 association constant $\left(K_{3}\right)$, the degradation rate constants of $\mathrm{T} 7$ mRNAs $\left(k_{\mathrm{dm}}\right)$ and T7 proteins $\left(k_{\mathrm{dp}}\right)$. Base values of these parameters were presented previously [31] and detailed in SOM.

To simulate mutants that carried multiple mutations, we generated $50000 \mathrm{~T} 7$ mutants that each had random values for 28 of the 30 parameters listed earlier. A random value was selected within the range from 0.1 to 10 times the base value of the corresponding parameter following a uniform distribution on a logarithmic scale. As the relative activities of promoters were used as weighting factors for distributing EcRNAPs or T7RNAPs, it is unnecessary to change all promoter activities simultaneously. For either the host or T7 RNAP, we kept one promoter activity constant while changing the others. Specifically, we held constant the relative activities of promoter 10 (for T7RNAP) and promoter A1 (for EcRNAP).

Because gene expression in $\mathrm{T} 7$ growth is coupled with transcription-mediated entry of the $\mathrm{T} 7$ genome [40], relocating 
a T7 genetic element within the genome can affect the timing of expression for some genes. To characterise this effect, we generated 100000 T7 mutants by randomly permuting the 72 internal elements of the T7 genome, and then characterised their growth. The permutation space is astronomically huge: the total number of possible permuted genomes is $72 ! \simeq 6 \times 10^{103}$, and the 100000 mutants accounted for only an infinitesimally small sampling of the entire space. Nevertheless, they seemed sufficient to give a representative view; the distribution of growth rates remained similar overall for sample sizes above 10000 .

\subsection{Host environments}

Each T7 mutant (generated by parametric or gene-order perturbation) was characterised in two contrasting hostresource environments: a finite environment and an infinite environment. As shown in Table 1, the host physiological parameters that define the finite environment correspond to a host cell growing at 1.0 doublings/h [36]. The infinite environment offers infinite levels of EcRNAPs, ribosomes, NTPs, amino acids and DNA contents in the cell (see Table S1 for more information and additional references).

\section{Results}

Overall, T7 seemed robust with respect to single-parameter perturbations (Fig. 1): all T7 mutants were viable in both environments in the presence of up to 10 -fold deviation for each of the selected parameters from its base value. Some mutants even grew faster than the wild-type, particularly in the infinite environment. For example, a decrease in the mRNA decay rate constant $\left(k_{\mathrm{dm}}\right)$ or an increase in the T7RNAP elongation rate $\left(k_{\mathrm{PT} 7}\right)$ caused a significant increase in the $\mathrm{T} 7$ growth rate (Fig. 1d). These results make intuitive sense. For example, a slower mRNA degradation would lead to overall faster mRNA accumulation, which in turn would lead to faster protein production, and eventually faster $\mathrm{T} 7$ progeny production.

Growth of 50000 randomly generated mutants, each of which contained 28 independent random mutations, yielded further evidence for the overall robustness of $\mathrm{T} 7$ with respect to its parameters (Fig. 2). All 50000 mutants were viable in the infinite environment and $99.95 \%$ (49 976) were viable in the finite environment. We defined growth rate normalised to the growth rate under base-case conditions. In the infinite environment, $\sim 24 \%$ (11983) of the mutants grew faster than the wild-type, with an average growth rate of 0.98 and a maximum growth rate of $\sim 44$. In contrast, only 5.3\% (2657) outperformed the wild-type in the finite environment. In this case, the average and the maximum growth rates were 0.51 and 1.7 , respectively, again highlighting the optimality of the wild-type relative to the mutants.

Table 1: Two host environments for T7 development

\begin{tabular}{lll}
\hline & Finite & Infinite \\
\hline Amino acid (residues) & $8.7 \times 10^{8}$ & $\infty$ \\
NTP (residues) & $5.5 \times 10^{7}$ & $\infty$ \\
DNA content (genome & 1.8 & $\infty$ \\
$\quad$ equivalents) & & \\
Ribosome & $1.08 \times 10^{4}$ & $\infty$ \\
RNAP & 503 & $\infty$ \\
\hline
\end{tabular}
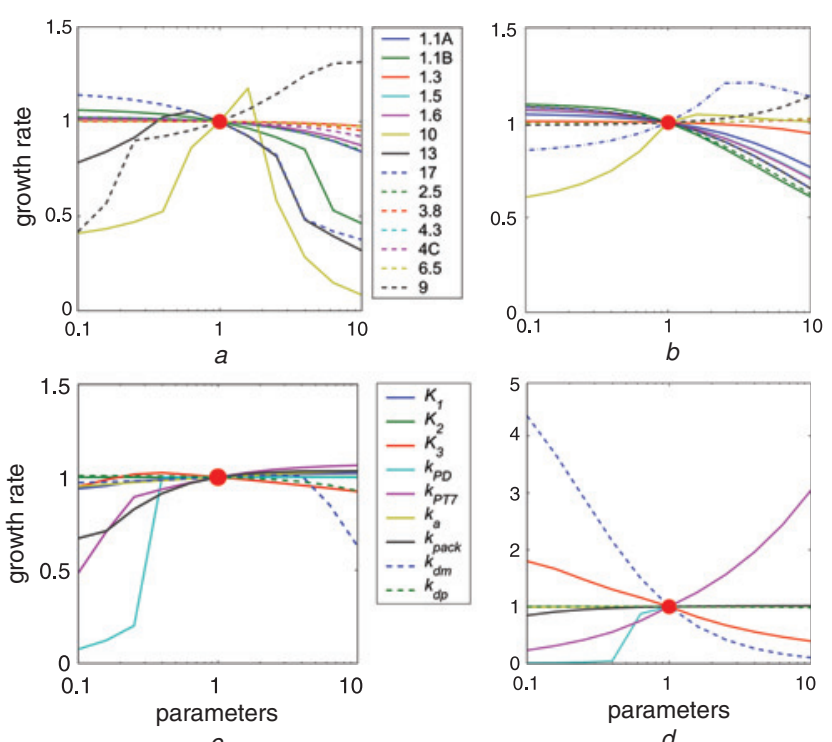

Fig. 1 The sensitivity of T7 growth rate to T7 promoter strengths $(a, b)$ and selected kinetic parameters $(c, d)$ for the finite host environment $(a, c)$ and the infinite host environment $(b, d)$

The $x$-axis represents parameters normalised with respect to their base-case values. The $y$-axes indicate the corresponding growth rate calculated for the chosen parameters. Growth rates were normalised with respect to the values calculated from the base-case parameter setting for either environment. Along each curve one parameter is varied while the others were kept constant. The base case is indicated by a filled circle

Compared with changes in kinetic parameters, random permutations of the genome organisation had much stronger, primarily deleterious effects on $\mathrm{T} 7$ growth (Fig. 3). More than $80 \%$ of $100000 \mathrm{~T} 7$ mutants with random genomes failed to grow in either the finite environment (83 663 dead) or the infinite environment (82 414 dead). In the finite environment, no mutants grew faster than the wild-type, and the maximum growth rate was only about $97 \%$ the wild-type value. In contrast, 4915 of the viable mutants in the infinite environment grew faster than the wild-type, with the maximum growth rate about 7 -fold greater than the wild-type value.

To gain insight into how random perturbations may affect T7 performance, we examined the efficiency of the 150000 $\mathrm{T} 7$ random mutants in balancing the production of viral proteins. For both types of random mutants, there was a stronger correlation between growth rates and efficiency in the finite environment than in the infinite environment (Figs. 4 and 5).
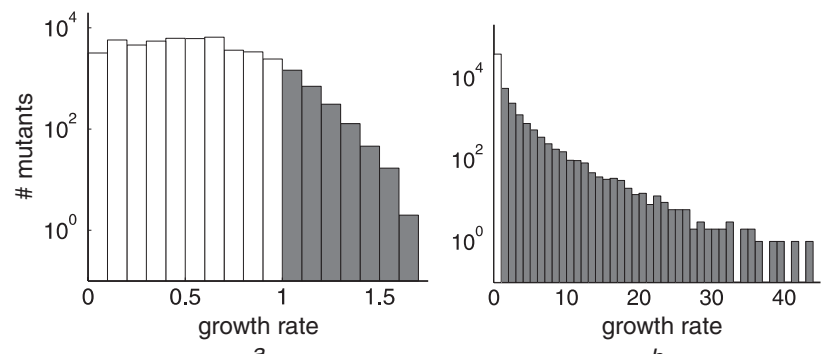

Fig. 2 Distribution of growth rates for 50000 T7 mutants, each with 28 independent random mutations

a Finite host environment

$b$ Infinite host environment

The mutants that grow faster than the wild-type are shown in the shaded part of the distribution 

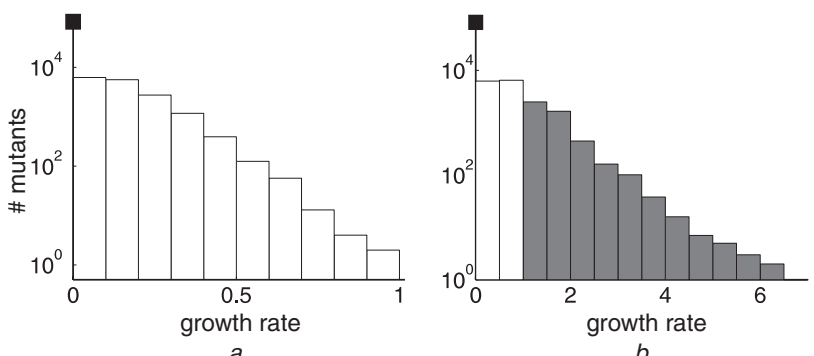

Fig. 3 Distribution of growth rates for 100000 mutants with randomly permutated genome

a Finite host environment

$b$ Infinite host environment

In both cases, the number of non-viable phage is indicated by a filled square at the zero-growth rate. The mutants that grow faster than wildtype $\mathrm{T} 7$ are shown in the shaded part of the distribution

\section{Discussion}

\subsection{Robustness and fragility}

Our results suggest that $\mathrm{T} 7$ is overall robust with respect to single or multiple perturbations in its parameters (Figs. 1 and 2). The vast majority of these $\mathrm{T} 7$ mutants are viable and many grew faster than the wild-type. In contrast, T7 is fragile to drastic perturbations in its genome structure: random permutations of the $\mathrm{T} 7$ genome are fatal in greater than $80 \%$ of the cases studied, regardless of the growth environment (Fig. 3). The detrimental effects are more pronounced in the finite environment, where none of the 100000 mutants can grow as fast as the wild-type. In

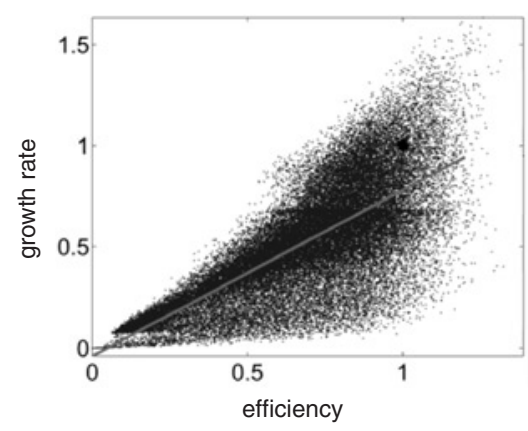

a

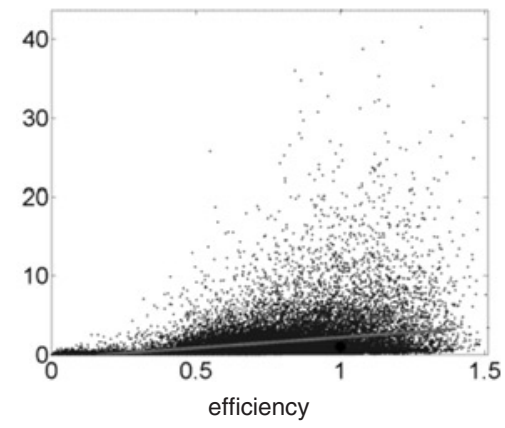

$b$

Fig. 4 Correlation of growth rates and efficiency for the 50000 mutants with random parameter values

$a$ Finite host environment

$b$ Infinite host environment

Each dot represents a mutant. Both efficiency and growth rates were normalised with respect to the corresponding wild-type values. A linear fit (indicated by lines) gives an $R^{2}$ value of 0.58 (finite environment) and 0.18 (infinite environment). The wild-type is indicated by a star asterisk symbol the infinite environment, however, about $5 \%$ of mutants grow faster than the wild-type, though the effect of random genome shuffling is overwhelmingly deleterious. Fragility of $\mathrm{T} 7$ with respect to random shuffling of genetic elements makes intuitive sense. T7 genome entry is mediated by EcRNAP after the first 850 bp enters the host cell [41, 42]. For a T7 mutant to survive, it requires at least one EcRNAP promoter within 850 bp of the entering end of the genome. With six EcRNAP promoters out of 72 internal genetic elements, there is a high probability to place all these promoters after $850 \mathrm{bp}$. The probability for these fatal configurations is approximately [(39 $937-850) /$ $39937]^{6} \simeq 88 \%$. This value agrees well with our simulation results $(\sim 82 \%)$. Therefore, failure to enter the cell may account for the failure for the majority of $\mathrm{T} 7$ random genomes to grow. If $\mathrm{T} 7$ enters the cell successfully, it may still fail to grow if at least one of its 23 essential genes is upstream of all promoters.

Parametric and genomic perturbations may be considered in terms of the extent of their impact on the underlying $\mathrm{T} 7$ reaction network. Most perturbations in single or multiple parameters cause quantitative rather than qualitative changes in the growth of the virus. They are in a sense 'anticipated' because biological mechanisms exist to create them - point mutations could result in changes in promoter activities or kinetic parameters of regulatory proteins. These changes can be buffered by the design of T7. For example, a major reason that single or multiple changes in promoter strengths caused little effects (Figs. 1 and 3) is likely because of redundancy of these promoters. Most T7 genes are transcribed from $15 \mathrm{~T} 7$ promoters (Fig. S1). If one or more of these 15 promoters (but not all) are inactivated, the remaining promoters still provide sufficient transcripts for $\mathrm{T} 7$ to survive (Fig. 1a).

In contrast, random perturbations of the $\mathrm{T} 7$ genome organisation may qualify as 'unanticipated' perturbations, because they disrupt the underlying structure of the T7 genetic network and such perturbations have not been found in naturally occurring phage. In this scenario, changes to $\mathrm{T} 7$ growth are no longer limited to the rates or the extent of some reactions (e.g. transcription of a gene). Instead, the structural disruption can drastically change temporal sequence of phage processes or prevent reactions that are essential for growth. For instance, a genomic mutant may fail to grow because all essential genes are promoter-less. The importance of the underlying network structure for proper functioning of a biological system is further illustrated by optimality of the wild-type T7 genome in the finite environment. For T7, the wild-type arrangement of the genetic elements will lead to efficient scheduling and timing of various cellular events involved in viral infection, which will, in turn, result in efficient use of resources provided in the finite environment (Fig. 5a) and thus nearly optimal growth.

Our predictions on fitness of shuffled T7 genomes can potentially be tested experimentally. For instance, recent work has generated a number of $\mathrm{T} 7$ variants with a few genes relocated to ectopic positions [37, 43]. Furthermore, progress has been made to 'refactor' the T7 genome so that it will be more amendable to large-scale rearrangement [44].

\subsection{Optimality and efficiency}

A distinct property of $\mathrm{T} 7$ is that growth of the wild-type is close to optimal in the finite environment. Relative to mutants, the wild-type T7 performs much better in the finite environment than in the infinite environment. 

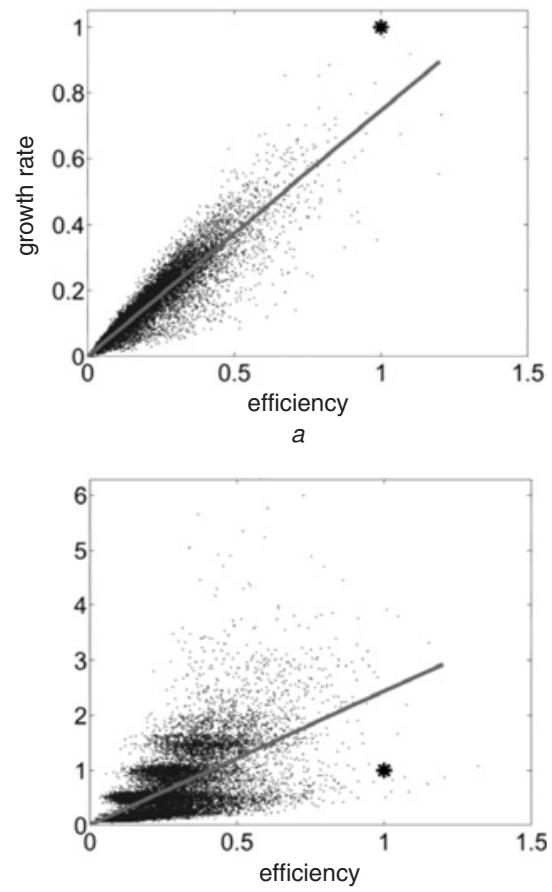

Fig. 5 Correlation of growth rates and efficiency for the 100000 mutants with randomly permuted genomes

$a$ Finite host environment

$b$ Infinite host environment

Each dot represents a mutant. Both efficiency and growth rates were normalised with respect to the corresponding wild-type values. A linear fit (indicated by lines) gives an $R^{2}$ value of 0.86 (finite environment) and 0.35 (infinite environment). The wild-type is indicated by a asterisk symbol

Regardless the type of mutations, most random mutants that outgrew the wild-type in the infinite environment grew much more slowly than the wild-type in the finite environment (Figs. 2 and 3). The optimality of wild-type T7 is particularly evident in its genomic structure: no mutants with random genomes grew faster than the wild-type in the finite environment, and only $\sim 5 \%$ did so in the infinite environment. Results from the previous study (Fig. 5) [37], where $2.8 \%$ of permutation mutants grew faster than the wild-type, are consistent with current observations. In the previous study, the host environment was a 'hybrid' of the two environments presented here: those simulations supplied infinite pools of nucleoside triphosphates, amino acids and ribosomes, but used finite levels of RNAPs (corresponding to a host growth rate of 1.0 doublings $/ \mathrm{h}$ ). The difference in growth environment and use of a different growth metrics may have caused differences in contours of fitness distributions. Despite these differences, however, an emerging picture from current and previous results is that faster growing mutants are more likely to occur with increasing amounts of resources.

Optimality of wild-type $\mathrm{T} 7$ in the finite environment probably results from its high efficiency in utilising limited resources. Figs. $4 a$ and $5 a$ indicate (a) the wildtype is more efficient than most mutants and (b) the fitness correlates well with efficiency in the finite environment (particularly for mutants with random genomes). The correlation makes intuitive sense. With limited resources, excessive production of some components will come at the expense of the others. This may reduce fitness if these other components are critical for growth. In the infinite environment, however, the phage is not penalised for being 'wasteful' in producing any of its products to achieve fast growth. Thus, growth rates do not correlate well with high efficiencies, as is evident in Figs. $4 b$ and $5 \mathrm{~b}$. In fact, many faster-growing mutants are much less efficient than the wild-type and would become less fit in the finite environment.

Our findings here, that phage can pay a fitness penalty for being inefficient, gives new perspective to previous results. Several gene 1 (encoding T7RNAP) ectopic mutants that put gene 1 under control of a T7RNAP promoter were predicted to grow faster than the wild-type, because of the formation of a positive feedback in the mutants where T7RNAP drives its own synthesis [37]. This prediction follows from the assumption that infinite resources were available, such that excessive T7RNAP production would not be detrimental to growth. Revised simulations using a host with finite resources, however, indicated that such ectopic mutants would grow more slowly than the wildtype: excessive synthesis of T7RNAP could lead to poor allocation of translation resources, resulting in a reduction in synthesis of $\mathrm{T} 7$ late proteins essential for progeny formation [45]. The new result is more consistent with experimental observation [37]. Similar behaviour has been observed in computational analysis of phase $\mathrm{Q} \beta$ infection cycle [39], which is found to be efficient in utilising energy.

Our analysis underscores the potential importance of the growth environment in shaping the design of an organism during evolution. Specifically, T7 relies on the host cell for its growth and a 'realistic' host cell provides finite resources. During evolution, T7 could have adapted for such finite environments, leading to high efficiency in utilising finite resources and overall optimality in growth rate. Consistent with this notion, further simulations indicate that wild-type T7 is nearly optimal in other realistic host environments corresponding to $E$. coli growth rates ranging from 0.5 to 2.0 doublings $/ \mathrm{h}$ (see Table $\mathrm{S} 1$ for dependence of host resources on the growth rate).

\subsection{Caveats}

Although wild-type T7 grows much faster and is more efficient in utilising resources than most mutants, it is not always the fastest or the most efficient, even in the finite environment (Figs. 1-3). Why do faster-growing and more efficient mutants appear? We suggest several mechanisms, which can be individually tested experimentally or computationally.

First, the host environment we adopted in this work represented a host cell growing under standard laboratory conditions [36] (see SOM for more details). Such a host cell is likely different from those growing in nature, and $\mathrm{T} 7$ could have been more optimised for naturally existing hosts. Consistent with this notion, recent work shows that the wild-type T7 can be quickly adapted to a new growth environment, resulting in increased fitness [43]. In addition, T7 may have evolved to be nearly optimal in different environments instead of being optimal in a single environment. That is, the wild-type $\mathrm{T} 7$ may be a 'generalist', whereas faster-growing mutants are 'specialists': they grow faster only under special conditions (e.g. in an environment with excessive resources). Further simulation results support this notion: the wild-type T7 is always close to optimal under a wide spectrum of realistic growth environments, corresponding to host cells with different growth rates (data not shown).

Secondly, the data and mechanisms incorporated in the model contain various degrees of uncertainty. In constructing the model, we have been faithful to the literature data 
and have not adjusted parameters to fit existing experimental data. However, potential uncertainties in the parameters may lead to biased representation of the 'wild-type'. The wild-type T7 defined in our model may be a variant, that is similar but not identical to the wild-type that the model aims to represent. In addition, we have assumed the two types of perturbations to be independent, although they may well be coupled, at least in laboratory conditions where T7 mutants were artificially evolved. A T7 mutant deleted for its lysin gene partially recovered its fitness by acquiring mutations in other genes [46]. Another T7 mutant with an ectopic RNAP gene moderately increased fitness by acquiring mutations in the early transcription terminator [43]. These examples indicate that perturbations to the genomic structure can be compensated by changes in kinetic parameters. With limited knowledge of the relationship between a gene sequence and its protein function, these types of interactions are difficult to anticipate or to implement in a kinetic model a priori.

Finally, as argued in the 'quasi-species' theory, a variant that is selected by evolution may not be the fittest; it can be sub-optimal and still win the race if it is 'supported' by its mutational neighbours with which it gives highest average fitness [47-49]. Our results seem to be consistent with this notion: most subtle mutations with respect to parameters or genomic structure lead to mutants with growth rates comparable to the wild-type value.

\section{Acknowledgments}

The work was supported by NSF EF-0313214 (award to J.Y.). We also thank three anonymous reviewers, whose comments helped to improve the clarity and presentation of our results.

\section{References}

1 Tyson, J.J., Chen, K., and Novak, B.: 'Network dynamics and cell physiology', Nat. Rev. Mol. Cell Biol., 2001, 2, (12), pp. 908-916

2 Endy, D., and Brent, R.: 'Modelling cellular behaviour', Nature, 2001, 409, (Suppl), pp. 391-395

3 Hasty, J., McMillen, D., Isaacs, F., and Collins, J.J.: 'Computational studies of gene regulatory networks: in numero molecular biology', Nat. Rev. Genet., 2001, 2, (4), pp. 268-279

4 Rao, C.V., and Arkin, A.P.: "Control motifs for intracellular regulatory networks', Annu. Rev. Biomed. Eng., 2001, 3, pp. 391-419

5 You, L.: 'Toward computational systems biology', Cell Biochem. Biophys., 2004, 40, (2), pp. 167-184

6 Iglesias, P.A., and Levchenko, A.: 'Modeling the cell's guidance system', Sci. STKE, 2002, 2002, (148), RE12

7 Wall, M.E., Hlavacek, W.S., and Savageau, M.A.: 'Design of gene circuits: lessons from bacteria', Nat. Rev. Genet., 2004, 5, (1), pp. 34-42

8 Kaern, M., Blake, W.J., and Collins, J.J.: 'The engineering of gene regulatory networks', Аnnu. Rev. Biomed. Eng., 2003, 5, pp. $179-206$

9 Asthagiri, A.R., and Lauffenburger, D.A.: 'Bioengineering models of cell signaling', Annu. Rev. Biomed. Eng., 2000, 2, pp. 31-53

10 Ideker, T., Galitski, T., and Hood, L.: 'A new approach to decoding life: systems biology', Annu. Rev. Genomics Hum. Genet., 2001, 2, pp. $343-372$

11 Stelling, J., Sauer, U., Szallasi, Z., Doyle, F.J. III, and Doyle, J.: 'Robustness of cellular functions', Cell, 2004, 118, (6), pp. 675-685

12 Barkai, N., and Leibler, S.: 'Robustness in simple biochemical networks', Nature, 1997, 387, (6636), pp. 913-917

13 Alon, U., Surette, M.G., Barkai, N., and Leibler, S.: 'Robustness in bacterial chemotaxis', Nature, 1999, 397, (6715), pp. 168-171

14 Yi, T.M., Huang, Y., Simon, M.I., and Doyle, J.: 'Robust perfect adaptation in bacterial chemotaxis through integral feedback control', Proc. Natl. Acad. Sci. USA, 2000, 97, (9), pp. 4649-4653

15 Morohashi, M., Winn, A.E., Borisuk, M.T., Bolouri, H., Doyle, J., and Kitano, H.: 'Robustness as a measure of plausibility in models of biochemical networks', J. Theor. Biol., 2002, 216, (1), pp. $19-30$
16 von Dassow, G., Meir, E., Munro, E.M., and Odell, G.M.: 'The segment polarity network is a robust developmental module', Nature, 2000, 406, (6792), pp. 188-192

17 Meir, E., von Dassow, G., Munro, E., and Odell, G.M.: 'Robustness, flexibility, and the role of lateral inhibition in the neurogenic network', Curr. Biol., 2002, 12, (10), pp. 778-786

18 Gonze, D., Halloy, J., and Goldbeter, A.: 'Robustness of circadian rhythms with respect to molecular noise', Proc. Natl. Acad. Sci. USA, 2002, 99, (2), pp. 673-678

19 Stelling, J., Gilles, E.D., and Doyle, F.J. III: 'Robustness properties of circadian clock architectures', Proc. Natl. Acad. Sci. USA, 2004, 101, (36), pp. 13210-13215

20 Forger, D.B., and Peskin, C.S.: 'Stochastic simulation of the mammalian circadian clock', Proc. Natl. Acad. Sci. USA, 2005, 102, (2), pp. 321-324

21 Mihalcescu, I., Hsing, W., and Leibler, S.: 'Resilient circadian oscillator revealed in individual cyanobacteria', Nature, 2004, 430, (6995), pp. 81-85

22 Kaern, M., Elston, T.C., Blake, W.J., and Collins, J.J.: 'Stochasticity in gene expression: from theories to phenotypes', Nat. Rev. Genet., 2005, 6, (6), pp. 451-464

23 Becskei, A., and Serrano, L.: 'Engineering stability in gene networks by autoregulation', Nature, 2000, 405, (6786), pp. 590-593

24 You, L., Cox, R.S. III, Weiss, R., and Arnold, F.H.: 'Programmed population control by cell-cell communication and regulated killing', Nature, 2004, 428, (6985), pp. 868-871

25 Balagadde, F.K., You, L., Hansen, C.L., Arnold, F.H., and Quake, S.R.: 'Long-term monitoring of bacteria undergoing programmed population control in a microchemostat', Science, 2005, 309, (5731), pp. 137-140

26 Elena, S.F., and Lenski, R.E.: 'Evolution experiments with microorganisms: the dynamics and genetic bases of adaptation', Nat. Rev. Genet., 2003, 4, (6), pp. 457-469

27 Mazer, S.J., and Damuth, J.: 'Evolutionary significance of variation' in Fox, C.W., Roff, D.A., and Fairbairn, D.J. (Eds.): 'Evolutionary ecology: concepts and case studies' (Oxford University Press, New York, NY, 2001), pp. 16-28

28 Savageau, M.A.: 'Genetic regulatory mechanisms and the ecological niche of Escherichia coli', Proc. Natl. Acad. Sci. USA, 1974, 71, (6), pp. 2453-2455

29 Savageau, M.A.: 'Design of molecular control mechanisms and the demand for gene expression', Proc. Natl. Acad. Sci. USA, 1977, 74, (12), pp. 5647-5651

30 Wagner, A.: 'Energy constraints on the evolution of gene expression', Mol. Biol. Evol., 2005, 22, (6), pp. 1365-1374

31 You, L., and Yin, J.: 'Dependence of epistasis on environment and mutation severity as revealed by in silico mutagenesis of phage T7', Genetics, 2002, 160, (4), pp. 1273-1281

32 Carlson, J.M., and Doyle, J.: 'Highly optimized tolerance: robustness and design in complex systems', Phys. Rev. Lett., 2000, 84, (11), pp. $2529-2532$

33 Carlson, J.M., and Doyle, J.: 'Complexity and robustness', Proc. Natl. Acad. Sci. USA, 2002, 99, (Suppl 1), pp. 2538-2545

34 Csete, M.E., and Doyle, J.C.: 'Reverse engineering of biological complexity', Science, 2002, 295, (5560), pp. 1664-1669

35 Endy, D., Kong, D., and Yin, J.: 'Intracellular kinetics of a growing virus: a genetically structured simulation for bacteriophage $\mathrm{T} 7$ ', Biotech. Bioeng., 1997, 55, pp. 375-389

36 You, L., Suthers, P.F., and Yin, J.: 'Effects of Escherichia coli physiology on growth of phage T7 in vivo and in silico', J. Bacteriol., 2002, 184, (7), pp. 1888-1894

37 Endy, D., You, L., Yin, J., and Molineux, I.J.: 'Computation, prediction, and experimental tests of fitness for bacteriophage $\mathrm{T} 7$ mutants with permuted genomes', Proc. Natl. Acad. Sci. USA, 2000, 97, (10), pp. 5375-5380

38 You, L.: 'The extension, application, and generalization of a phage T7 intracellular growth model [PhD]' (University of Wisconsin-Madison, Madison, WI, 2002)

39 Kim, H., and Yin, J.: 'Energy-efficient growth of phage $\mathrm{Q} \beta$ in Escherichia coli', Biotechnol. Bioeng., 2004, 88, (2), pp. 148-156

40 Studier, F.W., and Dunn, J.J.: 'Organization and expression of bacteriophage T7 DNA', CSH Quant. Biol., 1983, 47, pp. 999-1007

41 Garcia, L.R., and Molineux, I.J.: 'Rate of translocation of bacteriophage T7 DNA across the membranes of Escherichia coli, J. Bacteriol., 1995, 177, (14), pp. 4066-4076

42 Garcia, L.R., and Molineux, I.J.: 'Transcription-independent DNA translocation of bacteriophage T7 DNA into Escherichia coli', J. Bacteriol., 1996, 178, (23), pp. 6921-6929

43 Springman, R., Badgett, M.R., Molineux, I.J., and Bull, J.J.: 'Gene order constrains adaptation in bacteriophage T7', Virology, 2005, 341, (1), pp. 141-152

44 Chan, L.Y., Kosuri, S., and Endy, D.: 'Refactoring bacteriophage T7', Mol. Sys. Biol., 2005, 1, (1), pp. msb4100025-E1-msb4100025-E10 
45 You, L., and Yin, J.: 'Simulating the growth of viruses', Pac. Symp. Biocomput., 2001, pp. 532-543

46 Heineman, R.H., Molineux, I.J., and Bull, J.J.: 'Evolutionary robustness of an optimal phenotype: re-evolution of lysis in a bacteriophage deleted for its lysin gene', J. Mol. Evol., 2005, 61, (2), pp. 181-191
47 Eigen, M.: 'Molecular self-organization and the early stages of evolution', Q. Rev. Biophys., 1971, 4, (2), pp. 149-212

48 Eigen, M.: 'Selforganization of matter and the evolution of biological macromolecules', Naturwissenschaften, 1971, 58, (10), pp. 465-523

49 Wilke, C.O., Wang, J.L., Ofria, C., Lenski, R.E., and Adami, C. 'Evolution of digital organisms at high mutation rates leads to survival of the flattest', Nature, 2001, 412, (6844), pp. 331-333 